\title{
Four-wave Mixing of Topological Edge Plasmons in a Graphene Metasurface
}

\author{
Jian Wei You, Zhihao Lan, and Nicolae C. Panoiu \\ Department of Electronic and Electrical Engineering, University College London, Torrington Place, London WC1E 7JE, United Kingdom \\ Authore-mail address: j.you@ucl.ac.uk,z.lan@ucl.ac.uk, and n.panoiu@ucl.ac.uk.
}

\begin{abstract}
Using a specially engineered topological bandgap and strong third-order nonlinearity of certain graphene metasurfaces, we demonstrate that it is possible to achieve net optical gain in fourwave-mixing of topologically-protected plasmon edge modes. OCIS codes: $190.0190,190.4380,240.6680,160.3918$.
\end{abstract}

\section{Introduction}

Due to the unique properties of topological photonics, such as unidirectional mode propagation and defect immunization, this emerging field of photonics offers rich variety of potential applications to novel photonic devices, such as optical isolators [1], robust delay lines [2], and topological lasers [3]. To date, most of the studies in this area have been focused on linear topological photonic systems. However, unique topological features could also play an important role in the nonlinear regime, leading to novel collective phenomena such as new strongly-correlated states of light $[4,5]$. In this context, in this paper we demonstrate that specially designed graphene metasurfaces possess a topological bandgap and that, in certain circumstances, it is possible to achieve net optical gain in the four-wavemixing (FWM) of topologically protected plasmon edge modes that such graphene metasurfaces possess.

\section{Results and Discussion}

\subsection{Physical System and Linear Results}

The physical system investigated in this paper is illustrated in Fig. 1a: A graphene metasurface consisting of a hexagonal array of holes in a graphene sheet (Fig. 1b) is placed in a static magnetic field. Due to the time-reversalsymmetry breaking induced by the magneto-optical response of graphene in a static magnetic field, this physical system exhibits a topological plasmonic feature in a certain frequency bandgap, as per Fig. 1d and Fig. 1e. In order to gain more physical insights into the properties of bulk and edge plasmonic modes, the near field distribution of a finite graphene plasmonic metasurface was studied by using FEM simulations. In the case of bulk modes of the graphene metasurface (see Fig. 1f), the optical field spreads into the bulk region of this system. However, in the case of the edge mode (see Fig.1g), owing to topological protection, a unidirectional and defect-immune propagation of edge plasmons can be clearly observed along the edge of the graphene plasmonic metasurface.
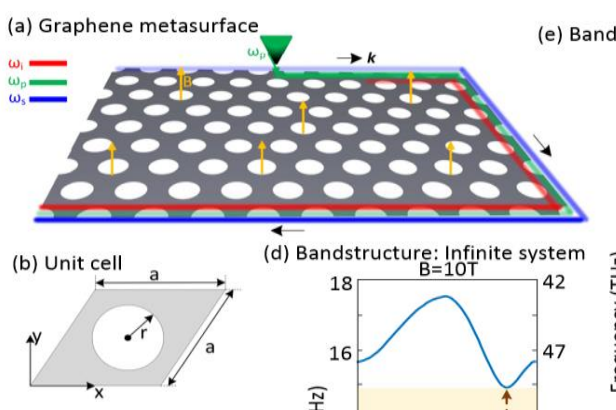

(c) Brillouin zone

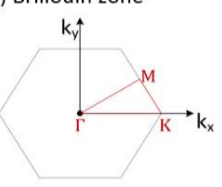

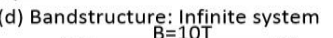

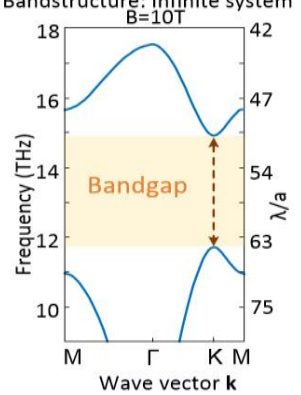

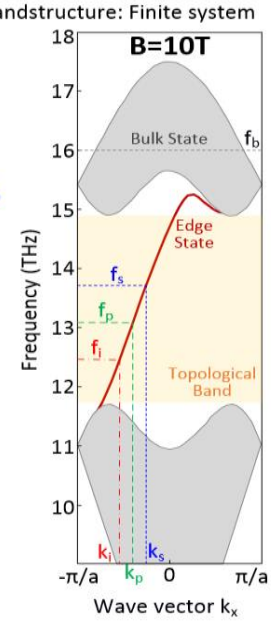
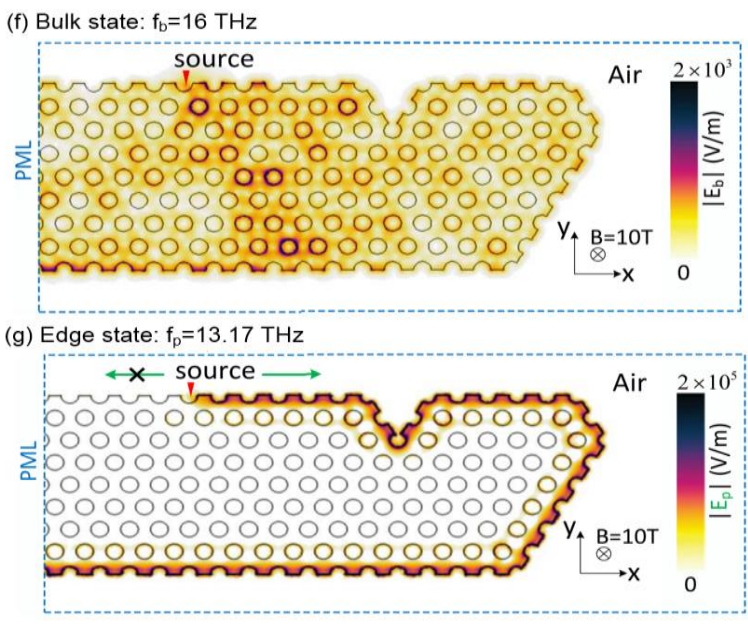

Fig. 1. (a) Four-wave mixing process in a topological graphene metasurface. (b), (c) Schematic of unit cell $(a=400 \mathrm{~nm}$ and $r=120 \mathrm{~nm}$ ) and first Brillouin zone, respectively. (d) Band diagram of an infinite graphene metasurface in a magnetic field of $B=10 \mathrm{~T}$; (e) Projected band diagram of a finite graphene metasurface with the red curve indicating the edge plasmon mode. (f), (g) Field profiles of a bulk and edge mode, respectively. 


\subsection{Four-wave-mixing Interaction of One-way Graphene Plasmon Modes}

As Fig.1e suggests, the topological bandgap is wide enough to allow one to readily sustain a degenerate FWM process for which all the interacting modes lie inside this bandgap. Moreover, to achieve a highly efficient energy conversion efficiency in a FWM process, the interacting optical waves should be phase-matched. In Fig 2a, we present the dispersion map of the FWM phase mismatch, $\Delta \phi$, the results being derived from the dispersion of the edge-state depicted in Fig.1e. This figure illustrates that both the frequency- and phase-matching conditions are satisfied at the pump frequency of $f_{p}=13.17 \mathrm{THz}$, signal frequency $f_{s}=13.72 \mathrm{THz}$, and idler frequency $f_{i}=12.62 \mathrm{THz}$. By employing nonlinear FEM simulations, we also demonstrated that it is possible that, when $\Delta \phi=0$, the FWM process leads to the amplification of the signal and the emergence of the idler at the predicted frequency. Importantly, since the pump, signal, and idler frequencies are inside the topological bandgap, as per Fig. 1e, all three interacting guided edge modes are topologically protected, thus they exhibit a unidirectional and defect-immune edge propagation. In particular, these conclusions are supported by the data presented in Fig. 2b and Fig. 2c.
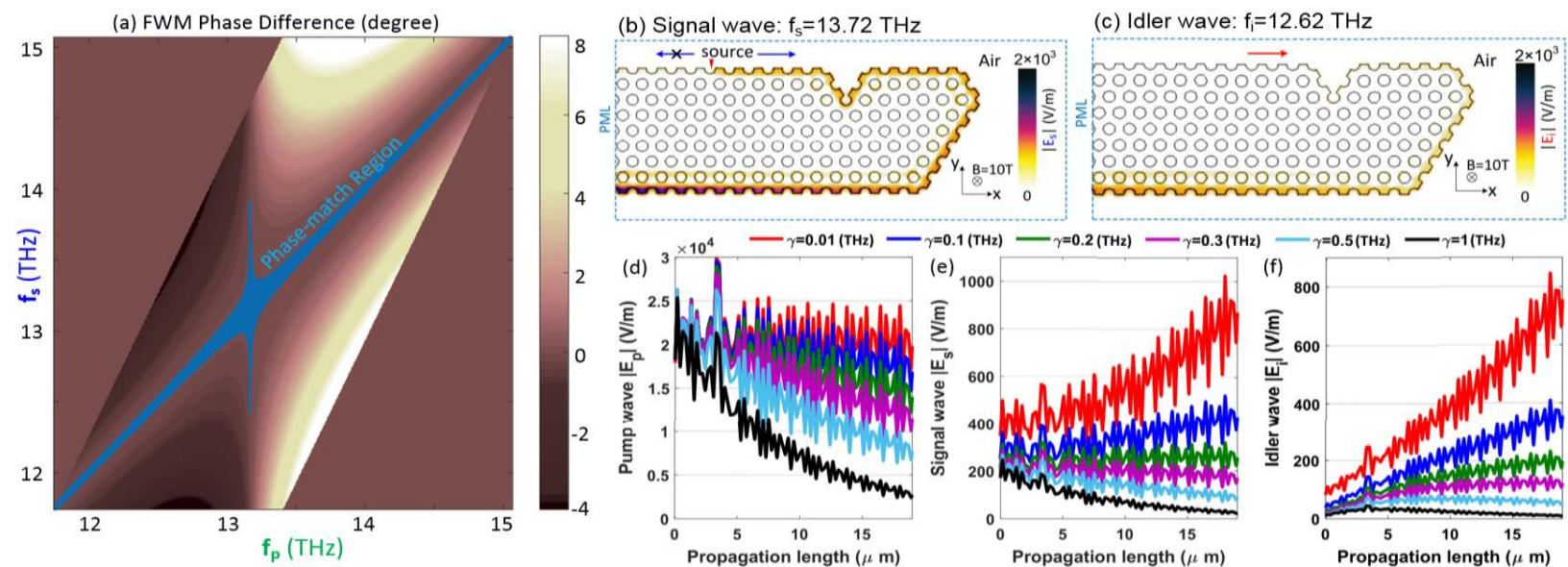

Fig. 2. (a) Dispersion map of the FWM phase mismatch $\Delta \phi$. The domain in blue corresponds to $\Delta \phi<10^{-3}$. (b), (c) Spatial profile of the signal and idler modes, respectively, demonstrating that both are amplified and topologically protected. Calculations are performed for $\Delta \phi=0$. (d), (e), and (f) Propagation of one-way edgemode pump, signal, and idler, respectively, determined for different plasmon damping rates, $\gamma$.

In practical applications, the intrinsic loss of graphene must be considered as it can significantly alter the performance of graphene-based devices. To this end, a one-way plasmon mode with nonzero damping rate $(\gamma \neq 0)$ is studied, the results being presented in Figs. 2d, 2e, and 2f. These figures show the electric field calculated along the edge of the finite graphene plasmonic system, at a distance of $80 \mathrm{~nm}$ above the surface of the graphene metasurface. These results demonstrate that a net gain can be achieved when $\gamma \leq 0.2 \mathrm{THz}$ [6]. The main reason why this important phenomenon occurs is that the energy of the pump wave could be effectively transferred to the signal at a rate larger than the rate at which the signal power dissipates. In other words, the intrinsic loss of the signal is compensated by the energy transferred from the pump mode to the signal via the FWM interaction.

\section{Conclusions}

In summary, we have demonstrated that certain specially engineered graphene metasurfaces possess a topological bandgap and one-way plasmon edge-mode, which can be employed to realize effective four-wave mixing. In particular, this graphene-based plasmonic system can be used to achieve net optical gain at deep-subwavelength scale.

\section{References}

[1] D. D. Solnyshkov, O. Bleu, and G. Malpuech, "Topological optical isolator based on polaritons graphene,” Appl. Phys. Lett. 112, 031106 (2018).

[2] X. Cheng, C. Jouvaud, X. Ni, S. H. Mousavi, A. Z. Genack, and A. B. Khanikaev, "Robust reconfigurable electromagnetic pathways within a photonic topological insulator," Nat. Mater. 15, 542-548 (2016).

[3] P. St-Jean, V. Goblot, E. Galopin, A. Lemaitre, T. Ozawa, L. Le Gratiet, I. Sagnes, J. Bloch, and A. Amo, "Lasing in topological edge states of a one-dimensional lattice," Nat. Photon. 11, 651-656 (2017).

[4] S. Mittal, E. A. Goldschmidt, and M. Hafezi, "A topological source of quantum light," Nature 561, 502-506 (2018).

[5] T. Ozawa, H. M. Price, A. Amo, N. Goldman, M. Hafezi, L. Lu, M. Rechtsman, D. Schuster, J. Simon, O. Zilberberg, and I. Carusotto, "Topological photonics," arXiv:1802.04173 (2018).

[6] J. W. You, Z. Lan, and N. C. Panoiu, "Four-wave mixing of topological edge plasmons in graphene metasurfaces," (to be submitted). 